\title{
Personalidade e lealdade: proposições de pesquisa com o modelo metateórico de motivação e personalidade
}

\section{(Personality and loyalty: research propositions with the motivation and personality meta- theoretical model)}

\author{
Kenny Basso \\ Lélis Balestrin Espartel \\ Cláudio Hoffmann Sampaio \\ Marcelo Gattermann Perin \\ Verner Luis Antoni
}

\begin{abstract}
Resumo
A personalidade, no estudo do comportamento do consumidor, tem-se mostrado um construto importante, mas pouco compreendido e utilizado nas pesquisas da área. Esse construto, enquanto preditor do comportamento, pode estar relacionado com a predisposição à lealdade em determinadas situações. Considerando a proposta de utilização da personalidade juntamente com variáveis comportamentais do indivíduo, este artigo, através do emprego da teoria dos traços de personalidade e do modelo metateórico de motivação e personalidade (modelo 3M) proposto por Mowen (2000), discute a possível ligação entre a personalidade do indivíduo e seu comportamento de lealdade. Para tanto, é feita uma revisão da literatura a respeito dos dois principais construtos (personalidade e lealdade), procurando evidenciar suas ligações, ao mesmo tempo em que são geradas proposições de pesquisa levando em conta as relações hierárquicas que possam existir entre esses construtos.
\end{abstract}

Palavras-chave: Personalidade; Comportamento do consumidor; Lealdade; Modelo 3M.

\begin{abstract}
Personality, in Consumer Behavior studies, has been an important construct, though little understood and rarely used in research. The construct, while predicting behavior, may be related with a predisposition to loyalty on certain situations. Considering the proposal of using the individual's personality together with behavioral variables, this article discusses a possible link between personality and loyalty behavior, through the use of the personality traits theory and the Motivation and Personality Meta-Theoretical Model (3M Model) proposed by Mowen (2000). For such, a literature review concerning the two main constructs (personality and loyalty) is carried out, aimed at highlighting their links. Proposals for research are presented, taking into account eventual hierarchical relations between those constructs.
\end{abstract}

Key words: Personality; Consumer behavior; Loyalty; 3M Model.

Conhecer o consumidor, seus comportamentos e seu processo decisório tem sido um dos principais desafios do marketing nos últimos tempos. Entender os processos de formação da lealdade e encontrar formas que permitam prever tal comportamento vem intrigando os pesquisadores da área. Nesse contexto, a personalidade, dentro da disciplina de Comportamento

Artigo recebido em 7 de abril de 2008 e aprovado para publicação em 10 de dezembro de 2008. 
do Consumidor, representa um construto relevante, mas notoriamente pouco compreendido e utilizado de forma marginal nas pesquisas da área, o que o torna suficientemente polêmico (BAUMGARTNER, 2002).

Por isso, estudar a personalidade no contexto de marketing tem sua relevância, já que ela parece responsável por um número significante de variâncias no comportamento e nas intenções do indivíduo (HARRIS; MOWEN, 2001). Blackwell et al. (2005) afirmam que variáveis de personalidade podem servir para diversos fins na área de marketing, indo desde o entendimento das preferências de um consumidor até a previsão de comportamentos frente a determinados estímulos, como a mídia.

Nesse sentido, a personalidade pode ser definida como uma organização única de fatores que caracterizam o indivíduo e determinam seu padrão de interação com o ambiente (HURLEY, 1998). A teoria dos traços de personalidade (PAUNONEN, 1998) vem sendo utilizada com maior assertividade na disciplina de Comportamento do Consumidor (BODEY; GRACE, 2006; HARRIS; MOWEN, 2001; HURLEY, 1998; LICATA et al., 2003; MONTEIRO; VEIGA, 2006; MOWEN, 2000; MOWEN; CARLSON, 2003; MOWEN; SPEARS, 1999). Essa teoria define que o indivíduo é formado por um conjunto de partes ou fatores básicos, que, quando agregados, formam a plenitude do ser. Hurley (1998) afirma que os traços de personalidade podem ser influenciados pelo meio e por atributos do indivíduo, expressos em diversas situações. Buss (1989) também sugere que a personalidade pode afetar as escolhas das pessoas e suas influências no meio.

Uma das maiores dificuldades para o uso da personalidade no marketing tem sido a sua adequação, visto que esse construto é amplamente discutido nas ciências psicológicas. Baumgartner (2002) afirma que as teorias de personalidade advindas da psicologia precisam de comprovações empíricas quanto à sua utilização na disciplina de Comportamento do Consumidor, a fim de reduzir os conflitos e as incertezas dos resultados esperados.

$\mathrm{Na}$ década de 1970, a linha de pesquisa relacionando personalidade com o comportamento do consumidor recebeu atenção de numerosos pesquisadores. Na maioria dos estudos as relações encontradas foram fracas ou até inexistentes. A causa disso pode estar nos dispositivos de mensuração utilizados, advindos da psicologia clínica, moldados para a identificação de transtornos e patologias (HARRIS; MOWEN, 2001; KASSARJIAN; SHEFFET, 1991; LICATA et al., 2003).

Em relação a essas pesquisas, a lealdade à marca foi um dos itens que compôs esses estudos (BAUMGARTNER, 2002; KASSARJIAN; SHEFFET, 1991). No entanto, os resultados mostraram-se pouco significantes para sustentar as afirmações, problema este relativo às formas 
de estudo e à precariedade dos procedimentos metodológicos empregados (BAUMGARTNER, 2002). A lealdade, por sua vez, é encarada não só como uma simples repetição de compra, mas como um processo que envolve um comprometimento e um engajamento muito mais profundo do indivíduo para com um produto ou marca (OLIVER, 1999). Dessa forma, pode-se aceitar que a lealdade seja formada por itens de cunho intrínseco ao indivíduo, relativos ao seu processo de aprendizagem e inerentes às causas comportamentais (SINGH, 1990). A personalidade, enquanto preditora do comportamento (CARVER; SCHEIER, 1990), pode estar relacionada com a predisposição à lealdade em determinadas situações. Porém, essas ligações podem representar padrões e papéis complexos no comportamento do indivíduo (HORTON, 1979).

Para minimizar o problema da mensuração da personalidade nos estudos de comportamento do consumidor, Mowen (2000) propôs um modelo hierárquico de análise composto por quatro níveis, para, a partir de traços de personalidade, predizer comportamentos dos indivíduos. Esse modelo vem sendo utilizado com sucesso em diversos estudos na área de marketing (HARRIS; MOWEN, 2001; LICATA et al., 2003; MONTEIRO; VEIGA, 2006; MOWEN, 2000; MOWEN; CARLSON, 2003). O modelo metateórico de motivação e personalidade (modelo 3M), como é denominado, define quatro níveis de traços que compõem a organização da personalidade: (1) traços elementares, (2) traços compostos, (3) traços situacionais e (4) traços superficiais. Os traços agem de tal forma a serem pontos de referência para a avaliação dos resultados, isto é, quando um resultado separa-se do seu ponto de referência, ocorre uma interrupção e o indivíduo é então motivado a trocar a programação de seu comportamento (CARVER; SCHEIER, 1990; MOWEN, 2000).

Seguindo essa linha, este artigo, no intuito de contribuir para a teoria em marketing, pretende gerar proposições acerca da relação preditiva da lealdade com uso de variáveis de personalidade por meio de uma adaptação do modelo 3M desenvolvido por Mowen (2000).

\section{Personalidade e teoria dos traços}

A personalidade pode ser entendida, segundo Davidoff (1983, p. 507), como “os padrões relativamente constantes e duradouros de perceber, pensar, sentir e comportar-se, os quais parecem dar às pessoas identidades separadas”. Essa definição permeia as motivações, os interesses, as atitudes, os pensamentos e as capacidades. A personalidade contém em seu escopo a ideia de que cada indivíduo possui a sua organização de personalidade, com características que são inerentes a tal; no entanto, ao se analisar diversos indivíduos, nota-se que determinados padrões emergem de características semelhantes. Assim, conhecer e entender a personalidade de um indivíduo pode estar ligado à predição do seu comportamento em uma determinada situação, 
tal como argumentam Pervin e John (2004, p. 23), para quem a personalidade "representa aquelas características da pessoa que explicam padrões consistentes de sentimentos, pensamentos e comportamentos".

Blackwell et al. (2005, p. 220) afirmam que a personalidade no estudo do consumo é "uma maquiagem individual única, que consistentemente influencia como a pessoa responde ao seu ambiente". Notando os diferentes padrões que podem emergir para um mesmo estímulo, a personalidade exerce um papel de direcionadora dos comportamentos.

Em uma definição contemporânea e adequada aos entendimentos do marketing e do comportamento do consumidor, Mowen (2000) afirma que a personalidade é um conjunto de construtos intrapsíquicos, hierarquicamente inter-relacionados, que revelam consistência através do tempo e que interagem com as diversas situações do contexto para influenciar os pensamentos, sentimentos e o comportamento dos indivíduos.

Uma vez que a personalidade possui diversos enfoques que podem ser alvo de estudo, o marketing tem se guiado pela corrente dos traços, que, de acordo com Mowen (2000), procura estudar as dimensões básicas da personalidade e tem se apresentado como promissora para essa área do conhecimento. Esse enfoque auxilia na construção de métricas adequadas à utilização no marketing, com ênfase no comportamento do consumidor.

A teoria dos traços de personalidade representa uma das diversas linhas de pesquisa dentro da personalidade (PERVIN; JOHN, 2004), encontrando na distinção entre os indivíduos formas de provocar a busca por padrões, relatando as diferenças e semelhanças do comportamento e o alcance destas. Nessa teoria, Buss (1989) define que os traços são homogêneos por natureza e percebidos como melhores preditores do comportamento.

Em adição à teoria dos traços, a teoria do controle também se encaixa no estudo da personalidade, uma vez que possui um valor explanatório significativo acerca da motivação humana. Embora a teoria do controle ofereça diversos esclarecimentos sobre o comportamento humano, Carver e Scheier (1990) definem que ela ainda possui uma baixa participação na área da personalidade. Os mesmos autores definem que as bases da teoria do controle são simples: o feedback negativo é o ponto central do mecanismo que regula o comportamento. Ela aceita que os comportamentos são guiados pela discrepância entre a posição atual em que o indivíduo se encontra e a posição de referência em que este deseja estar. Integram-se, desse modo, os conceitos de personalidade e motivação.

Não obstante, nota-se que a personalidade define as características inerentes à forma de ser do indivíduo, que por sua vez não estão dissociadas dos padrões e motivações para compra e 
posse dos produtos (TIAN; BELK, 2005). Essa relação é descrita em maior profundidade na seção a seguir.

\section{Personalidade e comportamento do consumidor}

Historicamente, o estudo da personalidade junto ao consumo teve início à época da Segunda Guerra Mundial, com a utilização das variáveis psicográficas para descrever a aparência das pessoas em um contexto de marketing, por meio de entendimentos psicológicos, sociológicos e antropológicos. A utilização dessas variáveis passou a ser adotada nos estudos, a fim de prover uma maior precisão para as decisões, tomadas com base em variáveis demográficas. Nisso, Kassarjian e Sheffet (1991) mostram as possíveis ligações entre a personalidade do indivíduo e o seu comportamento de compra, evidenciando a capacidade de usar a personalidade como preditor do comportamento. No mesmo ponto, Mowen e Spears (1999) descobriram que diferenças individuais na personalidade podem agir de forma a influenciar o comportamento do indivíduo em diferentes situações. Ligada ao comportamento do consumidor, a personalidade parece responsável por um número significante de variâncias no comportamento, nas intenções e nas decisões do indivíduo (HARRIS; MOWEN, 2001).

Muitos fatores podem afetar a tomada de decisão, pois as constituições individuais fazem com que os enfoques e os resultados sejam diferentes para cada indivíduo (BLACKWELL et al., 2005; JAAKKOLA, 2007). Característica única de cada ser, a personalidade tem sido considerada como um dos fatores capazes de intervir no processo de tomada de decisão (PERVIN; JOHN, 2004). Quanto ao comportamento pós-compra, verifica-se que na maioria dos modelos a personalidade tem sido frequentemente proposta como um importante antecedente (SINGH, 1990).

Entretanto, os estudos que buscaram relações entre a personalidade e o comportamento do consumidor têm encontrado resultados equivocados (KASSARJIAN; SHEFFET, 1991). Os resultados preditivos têm sido muito fracos, com variâncias de explicação entre $5 \%$ e 10\%. No entanto, esses resultados podem advir de um enfoque simplista e superficial das variáveis de personalidade, indicando que outras variáveis devam ser usadas para encontrar as relações entre o comportamento e a personalidade (BAUMGARTNER, 2002). Kassarjian e Sheffet (1991) afirmam que a utilização dos instrumentos provenientes da psicologia não possui adequação suficiente para funcionar como ferramenta de pesquisa na área de marketing. No mesmo raciocínio, Baumgartner (2002) explica que uma clara definição do termo "personalidade", quanto à sua profundidade e lateralidade, pode ter sido a carência encontrada em muitos modelos propostos para estudar a personalidade e o consumo. Mooradian e Olver (1997) também 
consideram que os enganos na junção de personalidade e consumo ocorrem por considerar um relacionamento direto entre ambos, sem a consideração de outras variáveis, tais como diferenças individuais, que podem constituir-se em mecanismos de intervenção.

Não obstante, diversos estudos foram feitos procurando encontrar relações entre a personalidade e muitos outros construtos de comportamento do consumidor. Harris e Mowen (2001) utilizaram o modelo 3M procurando relações entre a personalidade e as intenções de barganha e reclamação, enquanto Mowen e Carlson (2003) fizeram uso do mesmo modelo procurando explicações para o comportamento de superstição, mensurando diversas variáveis de resultado que poderiam sofrer impacto da superstição. Bodey e Grace (2006) utilizaram a personalidade para prover melhores bases para a segmentação de clientes, entre reclamantes e não-reclamantes. Em nível nacional, Monteiro e Veiga (2006) aplicaram o modelo 3M de Mowen (2000) com o intuito de prever hábitos de compra de artigos esportivos e de moda. De qualquer forma, vários estudos têm sido conduzidos procurando refinar a ligação entre a personalidade e a forma como o consumidor se comporta (MOORADIAN; OLVER, 1997; MOWEN; HARRIS, 1998; MOWEN; SPEARS, 1999; MOWEN et al., 2004; MOWEN et al., 2007). A personalidade também vem sendo aplicada em estudos que relacionam características dos empregados de fronteira com medidas de performance (LICATA et al., 2003; HURLEY, 1998)

Alguns pesquisadores afirmam que a personalidade pode ser definida através do uso dos produtos (SIRGY, 1982), uma vez que os bens podem ser vistos como uma forma de recompensa ou de comunicação com os outros. Ahuvia (2005) também encontrou que a procura e a compra de determinados bens estão relacionadas com a criação e a manutenção de identidade e de autoexpressão. Horton (1979), por sua vez, afirma que a personalidade pode ser um melhor preditor do comportamento em indivíduos com menos idade, os quais não têm suas estruturas bem formadas.

Bodey e Grace (2006) afirmam que os traços de personalidade podem, em alguns casos, ao invés de instigar o comportamento, reprimi-lo, fazendo com que os indivíduos não exprimam suas verdadeiras intenções, passando a agir de acordo com "pseudofiltros" ativados pelos traços de personalidade.

Assim como Oliver (1993) utilizou as emoções baseadas no consumo em complemento a enfoques cognitivos para predizer a satisfação, Mooradian e Olver (1997) consideram a personalidade como passível de utilização para o estudo de predição de processos de póscompra. Estudando mais especificamente a lealdade, Bove e Mitzifiris (2007) encontraram evidências que podem ligar predisposição à lealdade e traços de personalidade. 


\section{Lealdade}

A lealdade, enquanto alvo de vários estudos acadêmicos, é por diversas vezes considerada como um antecedente do desempenho organizacional (RUST et al., 2001).

Dick e Basu (1994), ainda em relação a retornos da lealdade, afirmam que, ao passo que os clientes encontram-se menos motivados a buscar alternativas ou substitutos, eles engajam-se em comunicações boca-a-boca positivas e têm uma menor propensão a serem persuadidos pelos concorrentes. Bodey e Grace (2006) também afirmam que entender as necessidades dos consumidores auxilia a prover uma maior lealdade.

Historicamente, a lealdade, enquanto variável relacional, teve seu desenvolvimento em função do impacto esperado na lucratividade da organização.

A lealdade pode ser definida, segundo Oliver (1999, p. 34), como:

um comprometimento profundo em comprar ou usar novamente um produto ou serviço, de forma consistente, para, no futuro, provocar compras repetidas da mesma marca ou empresa, mesmo que existam influências situacionais e esforços de marketing capazes de causar comportamento de mudança.

Com base na definição de Dick e Basu (1994), a lealdade constitui-se na proporção, sequência e probabilidade em que o consumidor irá comprar do mesmo fornecedor. De acordo com Uncles et al. (2003), a lealdade pode ser conceituada popularmente como uma atitude que direciona para o relacionamento com uma marca, expressa pelo comportamento e moderada por características individuais, circunstanciais e da situação de compra.

Para ser considerado leal, o consumidor deve possuir um comportamento de compra repetida e uma atitude positiva em relação ao produto ou organização, em comparação com as outras alternativas (CURASI; KENNEDY, 2002).

Segundo Espartel e Rossi (2006), os estudos de lealdade têm convergido para duas vertentes: uma que considera a existência da lealdade multimarcas (OLIVER, 1999), dando foco para facetas comportamentais; e outra que considera a lealdade mental, focando em percepções, atitudes e crenças. McMullan e Gilmore (2003) consideram que as duas principais linhas de pesquisa da lealdade abrangem, por um lado, as atitudes e, por outro, os comportamentos.

Para que seja possível detectar a lealdade verdadeira, é necessário avaliar as crenças, os afetos e as intenções do consumidor dentro de uma estrutura tradicional de atitude (OLIVER, 1999). Nisso, Vieira e Damacena (2007) relatam que as experiências, os conhecimentos, as crenças e as características únicas do indivíduo (personalidade) irão ter um impacto forte no comportamento de compra, auxiliando-o na tomada de decisão e na diferenciação entre lojas 
e/ou produtos. Assim, uma percepção prévia pode ser uma explicação sobre a tendência à lealdade.

A base da distinção entre a lealdade verdadeira e a recompra por inércia é a resposta comportamental não aleatória, função de um processo psicológico, ou como alguns autores apontam (p.ex. DICK; BASU, 1994), a atitude do consumidor em relação à marca. A lealdade a uma marca ou produto requer uma forte disposição interna do consumidor em continuar comprando a mesma marca (componente atitudinal). Por outro lado, o comportamento de inércia requer apenas um comportamento habitual do consumidor que busca reduzir os esforços físicos e mentais do processo de compra (AMINE, 1998).

O estudo dos antecedentes da lealdade revelou que tanto a satisfação quanto a confiança exercem um papel importante no processo de formação da lealdade (BOVE; MITZIFIRIS, 2007). A satisfação foi identificada como antecedente da lealdade por Zeithaml et al. (1996): quando um cliente está satisfeito com os produtos ou serviços ofertados pela organização, este permanece ligado à empresa por um tempo superior, implicando não só uma repetição de compra, mas um aumento dos volumes de compra e uma intenção favorável para com os produtos, serviços ou a empresa.

A satisfação pode ser definida como "uma resposta afetiva sumária de intensidade variável, com um ponto de determinação específico de tempo e duração limitada, direcionada a aspectos focais da compra e/ou consumo de um produto" (GIESE; COTE, 2000, p. 3).

Através do tempo alguns pesquisadores encontraram relações fortes entre a satisfação e a lealdade (OLIVER et al., 1997), enquanto outros identificaram que essa relação é, de fato, tênue e frágil (DICK; BASU, 1994; GARBARINO; JOHNSON, 1999). Coyne (1989, p. 73) afirma que "quando a satisfação figura acima de um certo patamar, a lealdade de recompra aumenta rapidamente. Em contraste, quando a satisfação sente-se abaixo, a lealdade do cliente declina em igual rapidez".

A confiança, por sua vez, é tratada como um produto da satisfação e como um antecedente da lealdade, uma vez que representa a integridade e a dignidade percebida por um parceiro no outro (GARBARINO; JOHNSON, 1999; MORGAN; HUNT, 1994; SIRDESHMUKH et al., 2002). Os julgamentos imparciais, baseados na qualidade do tratamento interpessoal que o cliente recebe durante o encontro de serviço, também desempenham seu papel na coprodução para geração de lealdade, através de influências nas atitudes e comportamentos do cliente (MASTERSON et al., 2000).

As definições de confiança remetem à área da psicologia, conduzindo à noção de engajamento de um indivíduo para com opiniões ou comportamentos esperados e observados em 
outrem, embora isso incorra em riscos e vulnerabilidades (ROUSSEAU et al., 1998). Morgan e Hunt (1994, p. 23) citam que a confiança existe "quando uma parte tem confiança na confiabilidade e integridade de seu parceiro de troca". No entanto, alguns autores afirmam que a confiança é relativa às expectativas presentes do consumidor quanto ao fornecedor de serviço, do qual é dependente, condizendo com a confiabilidade e a promessa do serviço a ser entregue (SIRDESHMUKH et al., 2002).

Ao usar os construtos de satisfação e confiança como antecedentes da lealdade, Bove e Mitzifiris (2007) estabeleceram que as relações entre os traços de personalidade mais abstratos e os construtos ocorrem de forma direta; porém, Mowen (2000) cita que o modelo 3M, com sua organização hierárquica de traços de personalidade, pode ser mais bem aplicado para predição de variáveis comportamentais, pois proporciona a mediação de outros traços menos abstratos e mais ligados ao comportamento. Com isso, na seção a seguir o modelo 3M é explicado e adequado ao estudo da lealdade, conforme as proposições apresentadas.

\section{Modelo 3M}

Mowen (2000), com o intuito de agrupar diversas teorias da psicologia e do marketing, realizou uma consistente análise metateórica para gerar uma união do construto da personalidade, propondo o modelo metateórico de motivação e personalidade, denominado de modelo 3M. Esse modelo foi criado para prover uma melhor explicação e previsão dos comportamentos do indivíduo, por meio de traços de personalidade.

O modelo 3M integra a teoria do controle (CARVER; SCHEIER, 1990), a teoria da psicologia evolucionária (BUSS, 1991) e a teoria dos traços hierárquicos (PAUNONEN, 1998), abrangendo também a proposição dos cinco traços de personalidade (McCRAE; COSTA, 1997).

Mowen (2000) afirma que o modelo 3M, por meio da personalidade, tem o intuito de atingir as três principais funções na disciplina de Comportamento do Consumidor: prever, explicar e controlar comportamentos. Do ponto de vista aplicado, Mowen (2000) argumenta que o modelo deve ser considerado bem-sucedido se for capaz de explicar e prever comportamentos com maior precisão do que os modelos propostos anteriormente, que fracassaram nesse ponto.

De acordo com um enfoque hierárquico da organização da personalidade, conforme proposto por Mowen (2000), os traços de personalidade são organizados em uma hierarquia de quatro níveis. Essa hierarquia inclui: (1) traços elementares, (2) traços compostos, (3) traços situacionais e (4) traços superficiais. Cada nível é unidimensional e a sequência acontece de um nível maior de abstração (traços elementares), para um nível mais concreto e específico (traços superficiais). Os traços serão mais bem detalhados nas seções seguintes. 
Licata et al. (2003) notaram que os resultados do seu estudo foram consistentes para suportar a noção de hierarquia na organização da personalidade, bem como evidenciar as combinações que podem aparecer mediando relacionamentos diretos, especialmente quando da inserção dos traços compostos no modelo. Pode-se afirmar que o modelo hierárquico (modelo 3M) explica uma maior variância no comportamento do que outros modelos (LICATA et al., 2003), como o modelo de cinco fatores proposto por McCrae e Costa (1997).

Uma vantagem em se usar um modelo hierárquico de personalidade é que efeitos indiretos de traços elementares, compostos e situacionais sobre os traços superficiais podem ser descobertos (MOWEN; CARLSON, 2003). Licata et al. (2003) também afirmam que o modelo 3M supõe que os traços poderão afetar variáveis de resultado (p.ex., número de compras de cliente em modelos de lealdade) quando em um modelo.

O modelo $3 \mathrm{M}$ pode tanto ser usado para a análise de comportamento do consumidor (HARRIS; MOWEN, 2001), quanto para a análise da personalidade ligada à performance de vendas, sendo que diferentes traços podem referir-se a diferentes pessoas, e comportamentos diferentes podem requerer dominância de um traço em detrimento de outro (LICATA et al., 2003)

\section{Traços elementares}

Os traços elementares são as disposições unidimensionais mais básicas da organização hierárquica da personalidade, e podem interagir com os traços compostos (MOWEN, 2000; LICATA et al., 2003). Esses traços podem ser conceituados como autoesquemas e analisados como níveis mais altos em uma estrutura de controle. São caracterizados por definirem os padrões de pensamento, sentimento e comportamento de um indivíduo (MOWEN, 2000), o que é evidente nas definições mais clássicas da personalidade.

Os traços elementares utilizados no modelo 3M são os seguintes: (1) amabilidade, (2) abertura à experiência, (3) extroversão, (4) consciência, (5) instabilidade emocional, (6) necessidade de recursos materiais, (7) necessidade de recursos físicos/corporais e (8) necessidade de excitação.

A maioria dos traços elementares é derivada da estrutura de cinco fatores (McCRAE; COSTA, 1997), bastante utilizada em estudos de personalidade, porém sob um foco muito mais psicológico para a identificação de transtornos (HARRIS; MOWEN, 2001). Dessa estrutura Mowen (2000) aproveitou os cinco fatores: (1) amabilidade, (2) abertura à experiência, (3) extroversão, (4) consciência e (5) instabilidade emocional. 
Em função dos padrões do comportamento humano e da disponibilidade dos recursos, Mowen (2000) considera que a (6) necessidade de recursos materiais (materialismo) e as (7) necessidades físicas ou corporais sejam traços elementares também. No entanto, nenhum desses sete traços descreve a propensão individual de provocar mudanças no ambiente (interno ou externo). Surge assim o traço de (8) necessidade de excitação, advindo da teoria do controle (CARVER; SCHEIER, 1990), considerando que o indivíduo modifica os ambientes na busca de estímulos mais adequados a si. Na mesma linha, Holbrook e Hirschman (1982) mostraram que a excitação exerce efeito na tomada de decisão quando em situações arriscadas.

Harris e Mowen (2001) ainda afirmam que o uso dos traços elementares provê ao pesquisador uma possibilidade de predizer os traços compostos e superficiais de determinado indivíduo.

\section{Traços compostos}

Os traços compostos ocupam o segundo nível da hierarquia da personalidade proposta por Mowen (2000), sendo sequenciais aos traços elementares. São conceituados como combinações unidimensionais dos traços elementares, e uma vez que inúmeras combinações entre os traços elementares são possíveis, podem ser vislumbrados inúmeros traços compostos. Por exemplo, Mowen (2000), em sua proposição do modelo 3M, estudou oito traços compostos: (1) necessidade de aprendizado, (2) necessidade de jogar, (3) necessidade de atividade, (4) necessidade de pensamento, (5) motivação efetiva, (6) orientação futura, (7) orientação para a tarefa e (8) orientação social.

Os traços compostos são de difícil operacionalização, devido ao alcance teórico esperado do traço e sua manifestação no indivíduo, que podem ser dissonantes (MOWEN, 2000). De qualquer forma, os traços compostos podem explicar um maior número de variações nos traços situacionais (o próximo nível) do que os traços elementares. Convém destacar que os traços compostos podem não conter elementos físicos derivados dos traços elementares que os formam. Mowen e Carlson (2003) fizeram uso de dois traços compostos: a necessidade de aprendizado e a autoeficácia, enquanto Harris e Mowen (2001) utilizaram o traço de percepção de valor.

A necessidade de aprendizado, por definição, enquadra o indivíduo em uma tendência de engajamento na busca por desafios intelectuais significativos e que lhe gerem prazer (MOWEN; CARLSON, 2003), enquanto a autoeficácia é conceituada como uma capacidade relativa de motivação intrínseca para organizar e agir de acordo com os recursos pessoais percebidos. Já a percepção de valor utilizada por Harris e Mowen (2001) é definida como uma busca por preços 
ou custos baixos na aquisição de um bem, em contrapartida a altos níveis de qualidade ou retorno obtidos, de acordo com a percepção do consumidor ou comprador.

\section{Evidências empíricas de estudos anteriores}

O trabalho de Mowen (2000) encontrou diversas evidências acerca das relações entre os traços elementares e compostos, relações que apareceram de forma exploratória, devido à ausência de estudos a priori. O autor descobriu que a instabilidade emocional, a extroversão/introversão, a necessidade de excitação, a necessidade de recursos materiais, a consciência e a necessidade de recursos corporais estão ligadas à autoeficácia. Do mesmo modo, a necessidade de aprendizado utilizada por Mowen (2000) como um traço composto foi precedida pelos seguintes traços elementares: abertura à experiência, consciência, instabilidade emocional, necessidade de recursos materiais e necessidade de excitação. Destaca-se que todas as relações citadas foram positivas.

No contexto brasileiro, Monteiro e Veiga (2006), estudando o modelo 3M, verificaram as relações existentes entre os traços elementares e compostos (autoeficácia e necessidade de aprendizado), encontrando que a autoeficácia possui relação significativa e positiva com a abertura à experiência, a consciência e a necessidade de excitação, enquanto a necessidade de aprendizado relacionou-se positivamente com a abertura à experiência, a consciência, a amabilidade e a necessidade de excitação.

Em observância ao estudo feito por Harris e Mowen (2001) com a utilização do modelo 3M, que não propunha nenhum relacionamento a formar o traço composto de percepção de valor, encontrou-se que a amabilidade e a abertura à experiência precedem esse traço.

\section{Traços situacionais}

Os traços situacionais são contextualizados como fatores unidimensionais que revelam relativos padrões de comportamento do indivíduo em um contexto ambiental. São provenientes de combinações dos traços elementares e compostos em situações ambientais. Em outras palavras, Licata et al. (2003) afirmam que são definidos como tendências resistentes para expressar padrões consistentes de comportamento dentro de um contexto situacional geral.

Esses traços proporcionam ao pesquisador uma maior categorização das personalidades, enquanto os traços elementares e compostos dão apenas o entendimento dos processos que determinam os comportamentos. Mowen e Carlson (2003) usaram como traços situacionais, por exemplo, o interesse por esportes e o interesse por artes e humanidade. 
As escalas de mensuração dos traços situacionais respondem por uma maior quantidade de variações no comportamento do que os traços elementares e compostos. Neste estudo propõese que tanto a confiança quanto a satisfação desempenhem o papel de traços situacionais.

Bove e Mitzifiris (2007) afirmam que poucas evidências empíricas são encontradas relacionando a personalidade com a confiança do indivíduo. No entanto, Roccas et al. (2002) encontraram evidências de que traços abstratos de personalidade podem relacionar-se com a benevolência, enquanto uma dimensão da confiança (SIRDESHMUKH et al., 2002). A confiança parece estar ligada à personalidade, ao passo que traços abstratos de consciência e instabilidade emocional demonstraram relacionamentos positivos quando analisados de forma direta, isto é, sem variáveis intervenientes (BOVE; MITZIFIRIS, 2007).

A satisfação, ao considerar o uso de cognição para comparar as expectativas e confirmálas ou não, possui ligações com a personalidade do indivíduo (MOORADIAN; OLVER, 1997). A satisfação é a avaliação de uma experiência específica de consumo, a qual pode envolver componentes emocionais (OLIVER, 1993), que por sua vez possuem disposições básicas centradas na personalidade (MOORADIAN; OLVER, 1997).

\section{Evidências empíricas de estudos anteriores}

Mowen (2000) encontrou uma forte correlação entre a necessidade de aprendizado e a necessidade de cognição, essa última definida como a capacidade de pensar e processar as informações. Tais informações podem relacionar-se com análises de discrepâncias entre as expectativas e a performance demonstrada pelo produto, e com a definição apresentada por Oliver (1999) de expectativa, como uma antecipação das consequências futuras baseadas em informações de experiências anteriores, ou fontes de informações externas.

Harris e Mowen (2001) fizeram uso do traço percepção de valor, seguindo uma vertente na qual o valor percebido é representado pela razão entre qualidade e preço. Oliver e Swan (1989) consideraram que o valor que os consumidores percebem em relação à sua aquisição tem um forte impacto em variáveis de pós-compra, tal como a satisfação. Lichtenstein et al. (1990) encontraram que a percepção do valor pode influenciar no comportamento de compra do indivíduo, sendo uma variável de cunho individual.

Em decorrência de estudos como o de Oliver (1993), que considera as emoções como parte da formação da satisfação, define-se que os encantamentos e superações de expectativas, com ou sem apelos sentimentais, podem conduzir o indivíduo a níveis de satisfação. No entanto, alguns pontos de vista emergem, considerando que os elementos cognitivos são importantes para os julgamentos das emoções. Nisso, Mowen (2000), ao trabalhar a autoeficácia, conduz para o 
entendimento dos controles internos do indivíduo e, dessa forma, para o processo de julgamento das emoções, tornando assim as expectativas mais adequadas à realidade.

A autoeficácia, como traço composto utilizado por Mowen e Carlson (2003), é relativa ao controle percebido pelo indivíduo sobre os recursos disponíveis e os resultados de seus atos (MOWEN, 2000). No mesmo sentido, Sirdeshmukh et al. (2002) demonstram que a competência, tanto do consumidor quanto da organização, em uma troca relacional, pode ter influência na confiança, de forma que o controle sobre os recursos e atos pode estar relacionado com a detenção de competência.

Em concordância com as evidências apresentadas por Sirdeshmukh et al. (2002) de que a confiança está relacionada com a criação e percepção de valor e de que o inverso também pode ser verdadeiro, a percepção de valor do consumidor pode gerar atitudes favoráveis para com a organização, levando-o a atingir níveis mais elevados de confiança. Dessa forma, a razão preçoqualidade, que retrata a percepção de valor usada por Harris e Mowen (2001), pode estar relacionada com a confiança, pois nas trocas relacionais os consumidores procuram aumentar o valor recebido, tendo por vezes que confiar no fornecedor.

Entende-se que a necessidade de aprendizado pode impactar o conhecimento entre os parceiros de troca ou relação, através do processamento e busca por informações de cada uma das partes (MOWEN, 2000), podendo levar o indivíduo a ter maiores níveis de confiança em um dado produto, serviço ou organização.

Com base no exposto e na aceitação histórica de que os dois construtos representam antecedentes verificados da lealdade (ESPARTEL; ROSSI, 2006) e se enquadram na definição conceitual dos traços situacionais, isto é, ocorrem em contextos específicos e como implicação de predisposições do indivíduo e de variáveis externas a este, verifica-se um possível relacionamento entre os traços compostos e situacionais, como descreve a proposição a seguir:

\section{P1: Os traços compostos do modelo 3M (necessidade de aprendizado, percepção de valor e autoeficácia) irão combinar-se aditivamente e de forma distinta a fim de formar os traços situacionais (satisfação e confiança).}

\section{Traços superficiais}

Os traços superficiais representam as disposições específicas que ocorrem na matriz de comportamento do indivíduo em uma dada situação. Os traços superficiais individuais são combinações dos traços elementares, compostos e situacionais, juntamente com os objetivos da pessoa (HARRIS; MOWEN, 2001; MOWEN; CARLSON, 2003). No contexto de marketing, 
esse traço pode representar uma predisposição individual para uma categoria de produto em particular ou para determinado comportamento de consumo.

Nisso, o modelo 3M apresenta-se como forma de identificar o comportamento mais específico e também o mais abstrato, à luz das características de personalidade individuais, através de uma organização essencialmente hierárquica de personalidade.

Mowen e Spears (1999) acreditam que os traços superficiais podem ser mais bem explicados através de uma organização hierárquica, identificando uma gama de traços básicos e mais abstratos que possam se combinar e explicar os traços superficiais, próximos ao comportamento, ligando assim a explicação de determinada ação do indivíduo em um determinado contexto. Harris e Mowen (2001) afirmam que tais traços podem ser centenas, devido ao grande número de combinações que podem ocorrer entre os traços mais abstratos.

De acordo com Endler e Rosenstein (1997), os traços superficiais podem prever comportamentos em determinadas situações em que os indivíduos se encontram ou irão se encontrar. Enquanto os traços elementares, compostos e situacionais motivam o comportamento, os traços superficiais representam as verdadeiras tendências comportamentais em determinadas situações. A medição dos traços superficiais tende a ser feita por escalas que mensurem a extensão e a frequência de determinado comportamento em um contexto específico (HARRIS; MOWEN, 2001), provendo, assim, uma melhor previsão do comportamento que pode ocorrer ou até mesmo das intenções comportamentais.

Além disso, os traços superficiais são os que mais se aproximam do comportamento expresso em um determinado contexto, representando disposições para se comportar durante o tempo (LICATA et al., 2003; MOWEN, 2000; MOWEN; SPEARS, 1999).

Em relação à lealdade, diversos modelos foram testados empiricamente, relacionando a lealdade com seus vários antecedentes (AGUSTIN; SINGH, 2002; GARBARINO; JOHNSON, 1999; SIRDESHMUKH et al., 2002). Garbarino e Johnson (1999) afirmam que, em trocas transacionais, a satisfação pode ser um caminho para a lealdade, enquanto em trocas relacionais a confiança desempenha esse papel. Em estudo utilizando os traços de personalidade, Bove e Mitzifiris (2007) encontraram que a confiança e a satisfação conduzem à lealdade, após sofrerem influência de traços de personalidade. Mooradian e Olver (1997) também consideram que a satisfação pode relacionar-se com a lealdade, após sofrer impacto de variáveis de personalidade.

A lealdade, por acontecer em um contexto específico, representando as preferências de compra (JACOBY; KYNER, 1973), que podem advir de experiências e aprendizados do indivíduo (MOWEN, 2000), e por estar intimamente ligada a comportamentos expressos (DICK; BASU, 1994), pode ser conceituada como um traço superficial no modelo 3M. 


\section{Evidências empíricas de estudos anteriores}

Espartel e Rossi (2006), em estudo longitudinal, identificaram que a satisfação possui um forte impacto na lealdade, evidenciando a importância da satisfação nos modelos de lealdade. Nesse ponto, as experiências satisfatórias podem impactar positivamente na lealdade.

Matos e Henrique (2006) identificam relação significativa entre satisfação e lealdade, com maior correlação em estudos que utilizaram produtos (em vez de serviços) como contexto, e menor quando o tipo de análise adotado foi a regressão múltipla em comparação com os que usaram equações estruturais. Da mesma forma, quanto maior a amostra, menor a média da correlação apresentada, e quanto maior a confiabilidade do constructo lealdade, maior a correlação verificada entre satisfação e lealdade.

Esses resultados, em conjunto com os estudos de Oliver (1999), confirmam que o cliente satisfeito é mais propenso à lealdade. Mooradian e Olver (1997) afirmam que a satisfação é influenciada por processos cognitivos, tendo também antecedentes emocionais, o que se relaciona com o entendimento da personalidade do indivíduo e suas possíveis implicações, uma vez que se trata de processos emocionais. Os mesmos autores consideram que, em proximidade à personalidade e às emoções manifestas pelo indivíduo, estão as categorias de personalidade como neurotismo e extroversão como preditores do afeto negativo e positivo, respectivamente. $\mathrm{O}$ afeto, de acordo com Oliver (1999), pode estar contido na formação da lealdade.

Bove e Mitzifiris (2007) não só verificaram que a satisfação e a confiança relacionam-se com a lealdade, como também citam que variáveis de personalidade podem auxiliar na predição da lealdade devido à proximidade do construto de personalidade para com as disposições para ação e padronização de comportamentos, tal como expressa o conceito de lealdade. Chaudhuri e Holbrook (2001) consideram que a confiança em uma determinada marca pode ter impacto em dimensões comportamentais e atitudinais da lealdade, determinando que a confiança é o ponto central dos relacionamentos de longa duração entre o cliente e a empresa.

A confiança pode levar à construção e à sustentação tanto de relacionamentos quanto da participação de mercado de uma empresa. No entanto, a crença de que a confiança pode ser transformada diretamente em lealdade é simplista e enganosa, ao passo que estudos revelam que a conversão da confiança em lealdade é complexa, envolvendo múltiplos processos que requerem o entendimento de como os construtos antecedentes fazem o consumidor ter confiança (SIRDESHMUKH et al., 2002).

Relacionando confiança e lealdade, Reichheld e Schefter (2000, p. 107) afirmam que, para "ganhar a lealdade de um cliente, você primeiro deve ganhar sua confiança". Alguns 

relacionamentos, sendo as principais consequências estudadas a lealdade e a cooperação (GARBARINO; JOHNSON, 1999). Mackintosh e Lockshin (1997) encontraram que confiança na marca foi um fator crítico para o surgimento da lealdade no contexto de varejo.

Dado o exposto e observando as evidências empíricas, a proposição abaixo é relevante ao estudo da lealdade com uso de variáveis de personalidade.

\section{P2: Os traços situacionais (satisfação e confiança) irão combinar-se aditivamente e de forma distinta a fim de formar o traço superficial (lealdade).}

As proposições, bem como os constructos envolvidos na construção teórica do modelo, ficam mais claros a partir da visualização da Figura 1.

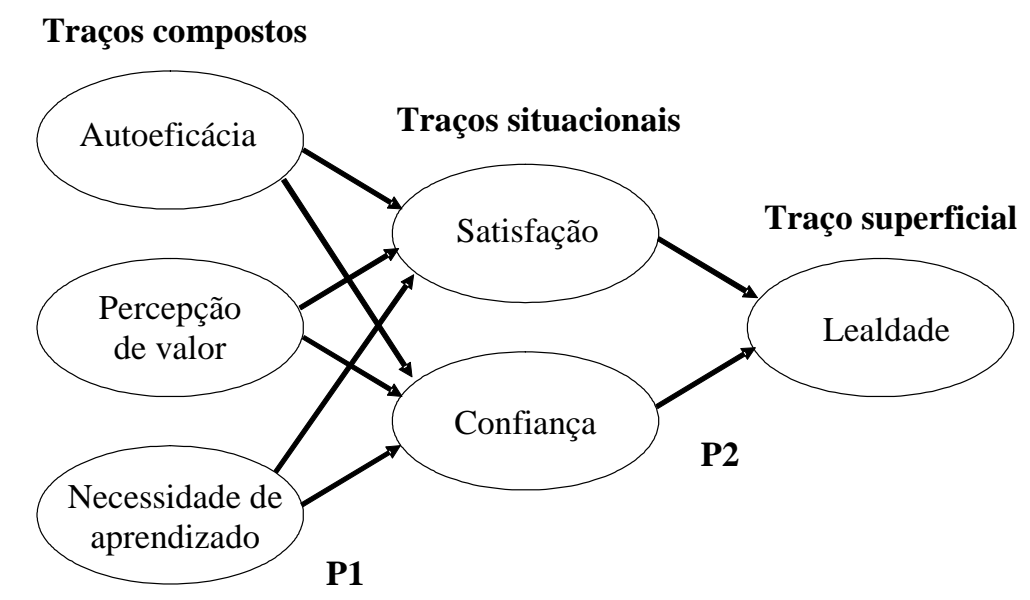

Figura 1 - Traços de personalidade e proposições de pesquisa.

Fonte: Elaborado pelos autores.

A proposta de relacionar a personalidade e a lealdade, por meio de um modelo hierárquico como demonstrado na Figura 1, é corroborada pela incessante procura por novas propostas de pesquisa que busquem uma relação significativa entre construtos psicológicos e construtos característicos da grande área de marketing, mais especificamente da disciplina de Comportamento do Consumidor. Desse modo, além de contribuir para o estudo de programas de relacionamento mais eficazes, a personalidade pode referenciar informações consistentes para que programas de lealdade sejam dirigidos com maior eficácia a diferentes segmentos de clientes. Para tanto, na aplicação do modelo proposto neste estudo espera-se que sejam elucidados construtos psicológicos de personalidade que atuem como preditores do comportamento de lealdade em determinados contextos. 


\section{Considerações finais}

Não é de admirar que não haja estudos recentes com resultados significativos sobre a relação entre personalidade e consumo, tamanha a aversão gerada por resultados pífios e tentativas falhas; no entanto, é clara a importância da personalidade e das pesquisas nesse campo, para que haja congruência da área e uma futura consolidação dos diversos fragmentos existentes (BAUMGARTNER, 2002; KASSARJIAN; SHEFFET, 1991; MOWEN, 2000).

Este estudo procurou elucidar uma possibilidade de integração dos construtos de lealdade e personalidade, provendo bases para estudos futuros através de mecanismos e construtos desenvolvidos para tal (KASSARJIAN; SHEFFET, 1991).

O modelo, com as relações propostas, deve ser testado através de estudos descritivos e experimentais, operacionalizados com consumidores de produtos e serviços em ambientes laboratoriais e de campo. Como técnica de análise sugere-se a utilização de modelagem de equações estruturais, buscando-se inferências sobre as relações diretas e indiretas entre os construtos presentes no modelo. Aliado a isso sugere-se que a análise da relação entre os traços compostos e os traços situacionais seja feita com o intuito de evidenciar os traços compostos que podem, aditivamente, contribuir para a formação dos traços situacionais, uma vez que a relação entre esses dois níveis hierárquicos deve ser comprovada empiricamente.

No âmbito do comportamento do consumidor, a personalidade pode seguir duas vias de utilização: a primeira teria um papel informacional para a segmentação; e a segunda seria uma forma de avaliar os interesses e expectativas dos consumidores, de forma preditiva. Esse segundo enfoque pode ser usado por profissionais no desenho de suas promoções, propagandas ou até mesmo do produto ofertado.

Desse modo, fica evidente que novos métodos de estudos da personalidade remetem a um entendimento mais profundo das características dos consumidores (BAUMGARTNER, 2002), fazendo com que as organizações possam ter suas estratégias e decisões mais focadas na lealdade e na manutenção de clientes no longo prazo (REICHHELD; SCHEFTER, 2000). Com isso, pode-se alcançar níveis em que os indivíduos demonstrem um determinado grau desejável de lealdade para a organização, fator considerado importante para o alcance de vantagens competitivas sustentáveis (DAY, 1994).

Os traços de personalidade (MOWEN, 2000), amparados por teorias de constituição de personalidade (BUSS, 1989) e por teorias de motivações e controle (CARVER; SCHEIER, 1990), podem conectar-se a construtos comportamentais do consumidor, como a lealdade (DICK; BASU, 1994; OLIVER, 1999), provendo para a disciplina de Comportamento do 
Consumidor um maior embasamento no que tange aos aspectos implícitos e inerentes às características individuais que norteiam as ações expressas em eventos de compra e consumo. A utilização de modelos hierárquicos pode ser um caminho para, em estudos futuros, encontrar-se as relações entre a personalidade e a lealdade, uma vez que essas relações não acontecem de forma direta (MOWEN, 2000).

Estudos futuros também podem enfatizar os contextos de inserção do indivíduo como atuantes sobre a possível relação entre a personalidade e o comportamento deste, uma vez que os traços de personalidade preconizados podem sofrer inferências do meio, potencializando seus efeitos sobre os traços de níveis mais elevados. Estudos longitudinais podem auxiliar a verificar as alterações dos traços em função do tempo, considerando os efeitos do amadurecimento e das modificações ambientais sobre a personalidade do indivíduo.

Por fim acredita-se que a personalidade possa prover entendimentos ainda mais profundos sobre o comportamento do consumidor, bem como gerar bases para a predição de comportamentos e, por conseguinte, estratégias e direcionamentos organizacionais mais precisos.

\section{Referências}

AGUSTIN, C.; SINGH, J. Satisfaction, trust, value and consumer loyalty: curvilinearities in relationship dynamics. In: CONFERENCE OF EUROPEAN MARKETING ACADEMY, 31, 2002, Lisboa. Proceedings... Portugal, 2002.

AHUVIA, A. C. Beyond the extended self: loved objects and consumers' identity narratives. Journal of Consumer Research, v. 32, n. 1, p.171-184, 2005.

AMINE, A. Consumers' true brand loyalty: the central role of commitment. Journal of Strategic Marketing, v. 6, p.305-319, 1998.

BAUMGARTNER. H. J. Toward a personology of the customer. Journal of Consumer Research, v. 29, n. 2, p. 286-292, 2002.

BLACKWELL, R.; MINIARD, P.; ENGEL, G. Comportamento do consumidor. 9. ed. São Paulo: Pioneira Thomson Learning, 2005.

BODEY, K.; GRACE, D. Segmenting service "complainers" and "non-complainers" on the basis of consumer characteristics. Journal of Services Marketing, v. 20, n. 3, p. 178-187, 2006.

BOVE, L.; MITZIFIRIS, B. Personality traits and the process of store loyalty in a transactional prone context. Journal of Services Marketing, v. 21, n. 7, p. 507-519, 2007.

BUSS, A. Personality as traits. American Psychologist, v. 44, n. 11, p. 1378-1388, 1989. 
BUSS, D. M. Evolutionary personality psychology. Annual Review of Psychology, v. 42, p. 459-491, 1991.

CARVER, C. S.; SCHEIER, M. F. Origins and functions of positive and negative affect: a control-process view. Psychological Review, v. 97, n. 1, p. 19-35, 1990.

CHAUDHURI, A.; HOLBROOK, M. B. The chain of effects from brand trust and brand affect to brand performance: the role of brand loyalty. Journal of Marketing, v. 65, n. 2, p. 81-93, 2001.

COYNE, K. P. Beyond services fads: meaningful strategies for the real world. Sloan Management Review, v. 30, n. 4, p. 69-76, Summer 1989.

CURASI, C. F.; KENNEDY, K. N. From prisoners to apostles: a typology of repeat buyers and loyal customers in service businesses. Journal of Services Marketing, v. 16, n. 4, p.322-341, 2002.

DAVIDOFF, L. Introdução à psicologia. São Paulo: McGraw-Hill, 1983.

DAY, G. S. The capabilities of market-driven organizations. Journal of Marketing, v. 58, p. 37-52, 1994.

DICK, A. S.; BASU, K. Customer loyalty: toward an integrated conceptual framework. Journal of the Academy of Marketing Science, v. 22, n.2, p.99-113, 1994.

ENDLER, N. S.; ROSENSTEIN, A. J. Evolution of the personality construct in marketing and its aplicability to contemporary personality research. Journal of Consumer Psychology, v. 6, p. 55-66, 1997.

ESPARTEL, L. B.; ROSSI, C. A. V. Um estudo longitudinal da lealdade do cliente e de seus antecedentes. In: ENCONTRO DE MARKETING, 2006, Rio de Janeiro. Anais... Rio de Janeiro: Anpad, 2006.

GARBARINO, E.; JOHNSON, M. S. The different roles of satisfaction, trust, and commitment in customer relationships. Journal of Marketing, v. 63, n. 2, p.70-87, 1999.

GIESE, J. L.; COTE, J. A. Defining consumer satisfaction. Academy of Marketing Science Review, v. 2000, n. 1, 2002. Disponível em: <http://www.amsreview.org/articles/giese012000.pdf>.

HARRIS, E. G.; MOWEN, J. C. The influence of cardinal-, central-, and surface-level personality traits on consumers' bargaining and complaint intentions. Psychology \& Marketing, v. 18, n. 11, p. 1155-1185, 2001.

HOLBROOK, M. B.; HIRSCHMAN, E. C. The experiential aspects of consumption: consumer fantasies, feelings and fun. Journal of Consumer Research, v. 9, p.132-140, 1982.

HORTON, R. L. Some relationships between personality and consumer decision making. Journal of Marketing Research, v. 16, n. 2, p. 233-246, 1979. 
HURLEY, R. F. Customer service behavior in retail settings: a study of the effect of service provider personality. Journal of the Academy of Marketing Science, v. 26, n. 2, p. 115-127, 1998.

JAAKKOLA, E. Purchase decision-making within professional consumer services: organizational or consumer buying behaviour? Marketing Theory, v. 7, n. 1, p. 93-108, 2007.

JACOBY, J.; KYNER, D. B. Brand loyalty vs. repeat purchasing behavior. Journal of Marketing Research, v. 10, n. 1, p. 1-9, 1973.

KASSARJIAN, H. H.; SHEFFET, M. J. Personality and consumer behavior: an update. In: KASSARJIAN, H.H, ROBERTSON, T.S (Ed.). Perspectives in consumer behavior. Englewood Cliffs: Prentice Hall, 1991. p. 281-303.

LICATA, J. W. et al. On the trait antecedents and outcomes of service worker job resourcefulness: a hierarchical model approach. Journal of the Academy of Marketing Science, v. 31, n. 3, p. 256-271, 2003.

LICHTENSTEIN, D. R.; NETEMEYER, R. G.; BURTON, S. Distinguishing coupon proneness from value consciousness: an acquisition-transaction utility theory perspective. Journal of Marketing, v. 54, p. 54-67, 1990.

MACKINTOSH, G.; LOCKSHIN, L. S. Retail relationships and store loyalty: a multi-level perspective. International Journal of Research in Marketing, v. 14, n. 5, p. 487-497, 1997.

MASTERSON, S. S.; LEWIS, K.; GOLDMAN, B. M.; TAYLOR, M. S. Integrating justice and social exchange: the differing effects of fair procedures and treatment on work relationships. Journal of the Academy of Management, v. 43, n. 4 p. 738-748, 2000.

MATOS, C. A.; HENRIQUE, J. L. Balanço do conhecimento em marketing: uma meta-análise dos resultados empíricos dos antecedentes e consequentes da satisfação e lealdade. In: ENCONTRO NACIONAL DA ANPAD, 30, 2006, Salvador. Anais... Rio de Janeiro: Anpad, 2006.

McCRAE, R. R; COSTA, P. T. Personality trait structure as a human universal. American Psychologist, v. 52, n. 5, p. 509-516, 1997.

McMULLAN, R.; GILMORE, A. The conceptual development of customer loyalty measurement: a proposed scale. Journal of Targeting, Measurement and Analysis for Marketing, v. 11, n. 3, p. 230-243, 2003.

MONTEIRO, P. R. R.; VEIGA, R. T. Personalidade e consumo: uma avaliação empírica do modelo metateórico de motivação e personalidade. In: ENCONTRO NACIONAL DA ANPAD, 30, 2006, Salvador. Anais... Rio de Janeiro: Anpad, 2006.

MOORADIAN, T. A.; OLVER, J. M. "I can't get no satisfaction": the impact of personality and emotion on postpurchase processes. Psychology \& Marketing, v. 14, n. 4, p. 379-393, 1997.

MORGAN, R.; HUNT, S. The commitment-trust theory of relationship marketing. Journal of Marketing, v. 58, n.3, p.20-38, 1994. 
MOWEN, J. C. The 3M model of motivation and personality: theory and empirical applications to consumer behavior. Boston: Kluer Academic Publishers, 2000.

MOWEN J. C.; CARLSON, B. Exploring the antecedents and consumer behavior consequences of the trait of superstition. Psychology \& Marketing, v. 20, n. 12, p. 1045-1065, 2003.

MOWEN, J. C.; HARRIS, E. G. Predicting consumer complaining and bargaining: a hierarchical approach using the $3 \mathrm{M}$ model of motivation and personality. American Marketing Association. Conference Proceedings, v. 9, p. 93-94, 1998.

MOWEN, J. C.; HARRIS, E. G.; BONE; S. A. Personality traits and fear response to print advertisements: theory and an empirical study. Psychology \& Marketing, v. 21, n. 11, p. 927943, 2004.

MOWEN, J. C.; SPEARS N. Understanding compulsive buying among college students: a hierarchical approach. Journal of Consumer Psychology, v. 8, p. 407-430, 1999.

MOWEN, J. C.; PARK, S.; ZABLAH, A. Toward a theory of motivation and personality with application to word-of-mouth communications. Journal of Business Research, v. 60, p. 590596, 2007.

OLIVER, R. L. Cognitive, affective, and attribution bases of the satisfaction response. Journal of Consumer Research, v. 20, p. 418-430, 1993.

OLIVER, R. L. Whence consumer loyalty? Journal of Marketing, v. 63, p. 33-44, 1999.

OLIVER, R. L.; RUST, R. T.; VARKI, S. Customer delight: foundations, findings, and managerial insight. Journal of Retailing, v. 73, n. 3, p. 311-336, 1997.

OLIVER, R. L.; SWAN, J. E. Consumer perceptions of interpersonal equity and satisfaction in transactions: a field survey approach. Journal of Marketing, v. 53, p.21-35, 1989.

PAUNONEN, S. V. Hierarchical organization of personality and prediction of behavior. Journal of Personality and Social Psychology, v. 74, p. 538-556, 1998.

PERVIN, L. A.; JOHN, O. P. Personalidade: teoria e pesquisa. 8. ed. Porto Alegre: Artmed, 2004.

REICHHELD, F. F.; SCHEFTER, P. E-loyalty: your secret weapon on the web. Harvard Business Review, v. 78, p. 105-113, 2000.

ROCCAS, S.; SAGIV, L.; SCHWARTZ, S. H.; KNAFO, A. The big five personality factors and personal values. Personality and Social Psychology Bulletin, v. 28, n. 6, p. 789-801, 2002.

ROUSSEAU, D.; SITKIN, S. B.; BURT, R.; CAMERER, C. Not so different after all: a crossdiscipline view of trust. The Academy of Management Review, v. 23, n. 3, p.1-12, 1998.

RUST, R. T.; ZEITHAML, V.; LEMON, K. N. O valor do cliente. Porto Alegre: Bookman, 2001. 
SINGH, J. A tipology of consumer dissatisfaction response styles. Journal of Retailing, v. 66, p. 57-99, 1990.

SIRDESHMUKH, D.; SINGH, J.; SABOL, B. Consumer trust, value, and loyalty in relational exchanges. Journal of Marketing, v. 66, n. 1, p.15-37, 2002.

SIRGY, M. J. Self-concept in consumer behavior: a critical review. Journal of Consumer Research, v. 9, p. 287-300, 1982.

TIAN, K.; BELK, R. W. Extended self and possesions in the workplace. Journal of Consumer Research, v. 21, n. 2, p.297-310, 2005.

UNCLES, M. D.; DOWLING, G. R.; HAMMOND, K. Customer loyalty and customer loyalty programs. Journal of Consumer Marketing, v. 20, n. 4, p. 294-317, 2003.

VIEIRA, V. A.; DAMACENA, C. Loyalty in the supermarket. Brazilian Administration Review, v. 4, n. 3, p. 47-62, 2007.

ZEITHAML, V. A.; BERRY, L. L.; PARASURAMAN, A. The behavioral consequences of service quality. Journal of Marketing, v. 60, n. 2, p. 31-46, 1996. 